\title{
Non-Canonical BAF Complex
}

National Cancer Institute

\section{Source}

National Cancer Institute. Non-Canonical BAF Complex. NCI Thesaurus. Code C156923.

A SWI/SNF family chromatin-remodeling complex comprised of least ACTB, BICRA (GLTSCR1) or BICRAL (GLTSCR1L), BRD9, SS18 or SS18L1/CREST, SMARCA2/BRM or SMARCA4/BRG1/BAF190A, ACT L6A/BAF53A or ACT L6B/BAF53B, SMARCC1/BAF155, and SMARCD1/BAF60A, SMARCD2/BAF60B or SMARCD3/BAF60C. 